\title{
A web-based decision support system for lower back pain diagnosis
}

\author{
Mohammed Arif Hasan Chowdhury \\ Head of the Department, Computer Science and Engineering, Cox's Bazar International University,Bangladesh
}

\begin{abstract}
In the era of science and technology, computer is the most spirited constituent in our today's health care sector with variety of facilities. The use of Artificial Intelligent (AI), Knowledge Management, is the vital components and demanding for knowledge and evidence based health care sector. Decision support System has the feasible to diagnosis the way professionals' delicacy patients for the better. In this paper, we efforts on the features of clinical decision support system for lower Back Pain (LBP) diagnosis. The objective of study is to develop a Web-based CDSS to support to diagnose and manage lower back pain according to guidelines. This paper will analyze how the decision support system efficiently work on LBP diagnosis and make it more efficient for the users.In this paper, we have studied the traditional LBP diagnosis system and web-based LBP system for the use of modern health care support. The research conducted with qualitative analysis in resulted through the interviewing with LBP patient and health care professionals on their view about the usage of web-based system in their diagnosis in which decision support system can play a role. It's focused on the development and evaluation of WDSS for diagnose and advising patient in lower back pain (LBP).
\end{abstract}

Keywords: Clinical decision support systems (CDSS), Lower back pain (LBP), Web-based decision support systems (WDSS).

\section{Introduction}

Information technology have inaugurated to be used in the arena of health as in many years and have delivered a lot of suitability ${ }^{8}$. Clinical Decision Support Systems (CDSS) which are accomplished of managing and assisting about clinical issues are growing day after dramatically. The studies linking the use of Decision Support Systems in the health arena initiated to seem in articles in the 1950s firstly. Then, the systems designed at diagnosing of diseases began to importance, and CDSSs became manifest. CDSSs are defined as a computer software supporting for decision making of health-care workers about clinical issues ${ }^{9}$. In addition with evolution of advanced technology, especially the World-Wide Web technologies, have twisted many prospects for research about Decision Support Systems. The technology of DSS is developing promptly and academic researchers need to catch-up with practitioners who are implementing innovative DSS ${ }^{7}$. Recently, CDSS was pooled with health request accomplished with telecommunication technologies, which have become the focus of considerable attention. For In terms of coordinating the outputs of workflow and managing the database of a core decision support system a complete work process engine was used ${ }^{10}$.

LBP is one of the most common reasons for visiting in the ambulatory care services ${ }^{1}$. In addition, LBP affects approximately $80 \%$ of adults at some time in life and occurs in all ages ${ }^{2}$. Low back pain is possibly from obesity and sedentary lifestyles ${ }^{3}$. Nowadays, the number of patients who suffers from LBP in increasing, so clinician takes longer of examination for preliminary screening until final diagnosis. Medical and clinical decision making require the clinician to apply accumulated knowledge for a specific amount of patients information to produce a result, that may be a diagnosis, prognosis, therapy or selection of proper therapeutic ${ }^{4}$. It is challenging for LBP diagnosing due to it involves a special knowledge which is related to anatomical and physiological structure, including clinical aspects ${ }^{5}$. Therefore, It leads to a big need for more accurate diagnosis (decision support systems) in patients with low back pain. So, the ultimate goal of the system is to help the clinicians, physical therapists and the pain specialists to reduce the uncertainty and accurate diagnosis of low back pain (LBP) patients in addition to improve diagnosis quality and timely knowledge access.

\section{Decision Support System}

Decision Support Systems(DSS) can be defined as computer technology solutions that can be used to support complex decision making and problem solving (Shim et al.)11. In terms of problems complexity and uncertainty, the DSS as a set of computer-based tools that deliver decision maker with interactive proficiencies. Decision Support Systems (DSSs) are interactive computer-based systems envisioned to assistance decision makers to exploit data and models to categorize and solve problems and make decisions 12. . DSSs help decision maker's use and manipulate data, apply checklists and heuristics, and build and use mathematical models. According to Turban13, a DSS has four major characteristics: it incorporates both data and models; it is designed to assist managers in their decision processes in semi structured (or unstructured) tasks; it supports, 
rather than replaces, managerial judgment; and its objective is to improve the effectiveness of decisions, not the efficiency with which decisions are being made.

DSScombine data, sophisticated analytical modelsandtools, and user-friendly software intoa single powerful system thatcan support semi- structuredand unstructured decision making ${ }^{13,14,15}$. The key mechanisms of theDSSaretheDSS database, the userinterface,andtheDSS software system (Figure 2). TheDSS databaseisagroup ofexisting data froma number ofapplications and groups. TheDSS userinterface allows informal interfacesamongst users of the systemandtheDSS software tools.

TheDSS software comprehendsthe software toolsthatare used for data analysis. It maycontain various Online Analytical Processing (OLAP) tools, data mining tools, or a collection of mathematicalandanalytical models that easilycan be madeaccessibletotheDSS users.

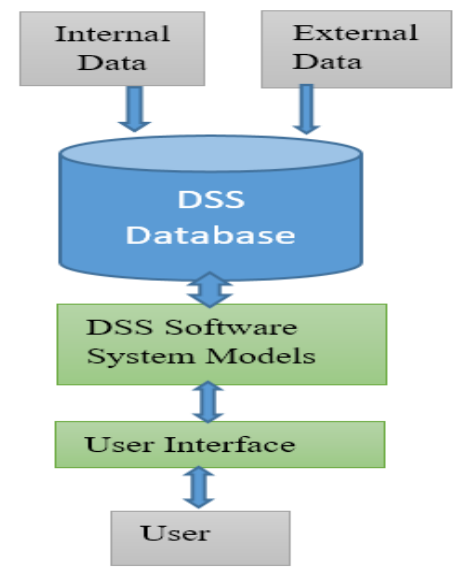

Figure 1: Main Components of DSS

\section{Web-based Decision Support System}

Web-based technologies are installed to expand the size of decision support systems concluded decision models, On-line Analysis Processing (OLAP) and data mining tools. All type of decision support related operations are accomplished on a network server in order to value from platform independence. Webbased DSS, decision-makers can segment or share open decision modules on the Internet by using identical protocols such as HTTP, and a standardized format like XML. The exodus about web based DSS denotes a shift from DSS generators (that allow users to develop specific applications characterized by limited deployment, inflexibility) to integrated cross application orientations that emphasize the reuse of applications and components. Knowledge bases and knowledge processing techniques can be used by deploying the web proficiencies in decision system. The use of web tools are reshaping the description of relations between information components and decision modules in a way that affects both the physical and logical design of the DSS, model visualization, scalability of decision modules and the development life cycle of DSSAs a result, the underlying architecture for Web-based DSS has moved from mainframes, to client-server systems, to Web and network technology based distributed systems that enable the integration of large amounts of data and decisions support tools originating from heterogeneous multidisciplinary sources for the provision of value-added information using knowledge discovery and data mining tools.

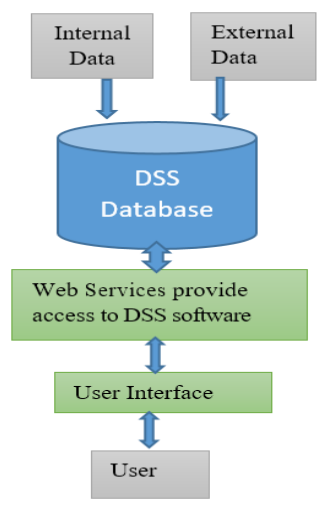

Figure 2: Web-Enabled DSS 


\section{Traditional LBP diagnosis}

First, when patients with low back pain are visiting a hospital, so the clinicians such as physicians, therapists normally perform a common process for LBP diagnosis and management ${ }^{5}$. Using a SOAP approach (subjective, objective tests, assessment, and patient management and planning), details are as follows:

Phase 1.Subjective testing, clinician collects relevant patient history and clinical information (question and answer) and gathers previous patient medical problems and treatment. Afterward, generates a preliminary diagnosis ${ }^{5}$.

Phase 2.Objective testing, clinician performs physical examinations to confirm/refine his preliminary diagnosis. Perhaps, clinician requires additional question and answer in case preliminary diagnosis is not supported ${ }^{5}$.

Phase 3. Assessment, clinician evaluates the pain, severity level and therapeutic protocols ${ }^{5}$.

Phase 4. Patient management planning, the clinician selects an appropriate therapeutic protocol and administers it to the patient until the satisfaction of patient recovery ${ }^{5}$

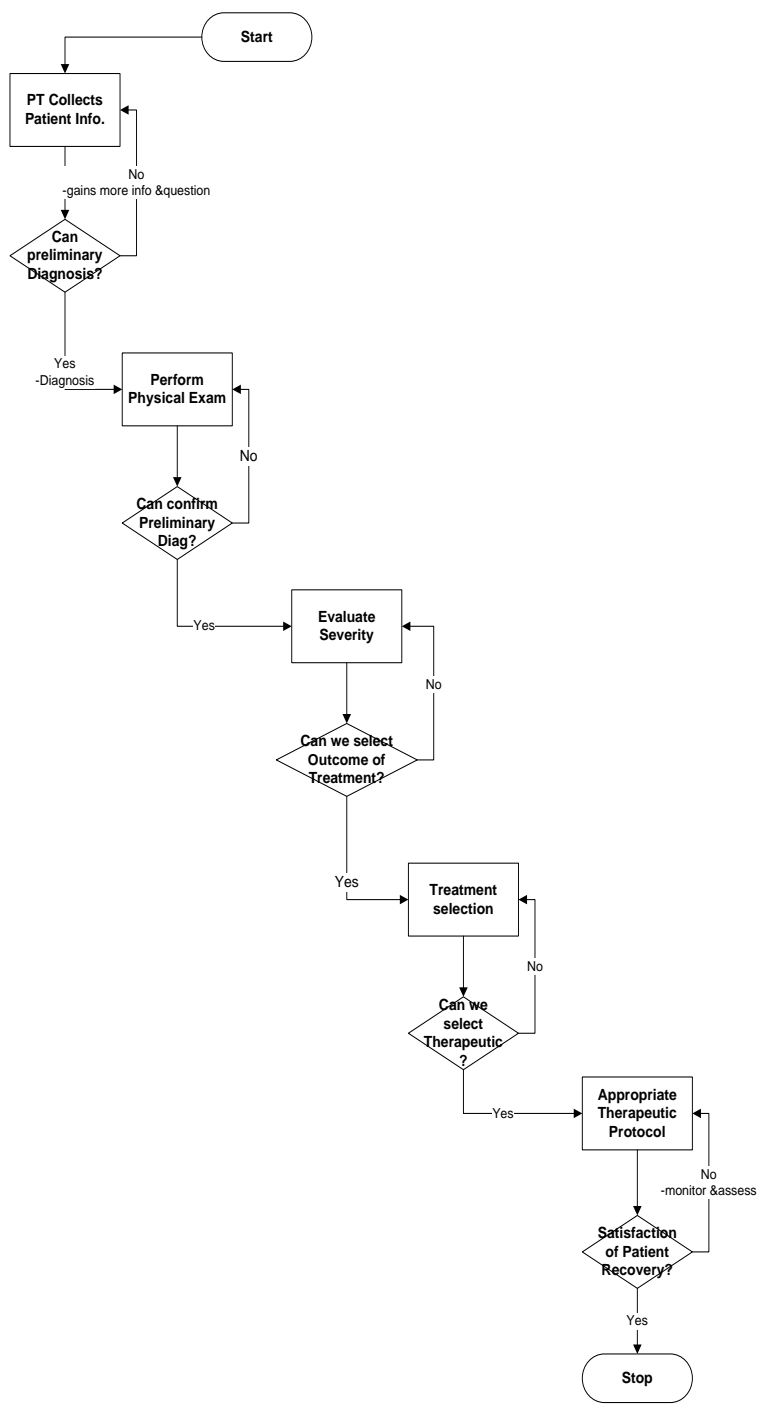

Figure. 3 The traditional process to diagnosis LBP

\section{Web-Based DSS for LBP diagnosis}

The web-based LBP diagnosis support system include the real-world cases that will provide the clinical efficacy by using targeted clinicians. It also included with knowledge base verification and system validation. The web-based system help the therapist to diagnosis LBP patients by using the modern technology which is easy to handle, reduce the uncertainty, take less time and provide more accurate decision. For this we have designed, implemented, and clinically evaluated a web-based decision support system ${ }^{5}$. In our system we have used some predefine questions for the patients, which is provided by the domain experts that can reduce the problem analysis, uncertainty modeling and reasoning as compare to other diagnosis methods for the LBP 
patients $^{5}$. Our proposed web-based system have already used 180 real-world cases that was collected from multiple, geographically dispersed clinics which provide the overall assessment of our evaluation design knowledge base verification, system validation and clinical efficacy.

\section{Design and technical reflection}

This system architecture is flexible and easy to use for the LBP patients as well as the physicians. It consists of a diagnostic interface, an inference engine, a case repository, a knowledge base and a central database. We have also some web-based interface to access the Internet. We have used 140 rules in our knowledge base database and to implement this we have used MySQL that uses a modified certainty factor scheme designed to alleviate the burdensome expert probability estimation requirements. The rules were provided by the highly experienced physical therapists and they are stored in the relational database. The inference engine that we have used in our web-based system is implemented by ANSI standard java 2 that can support multi-part diagnoses. We have a strong algorithm, which is running in the backend of the system, and it cans effectively addressing the exclusive negation characteristic of an LBP diagnosis. For each patient the system can provide a diagnosis which varying certainty values based on patients provided information and clinical evidence. It will also provide the intermediate results from the systems rule activation. The system will recommend diagnosis include the medical condition like what kind of diseases or abnormalities the patient have

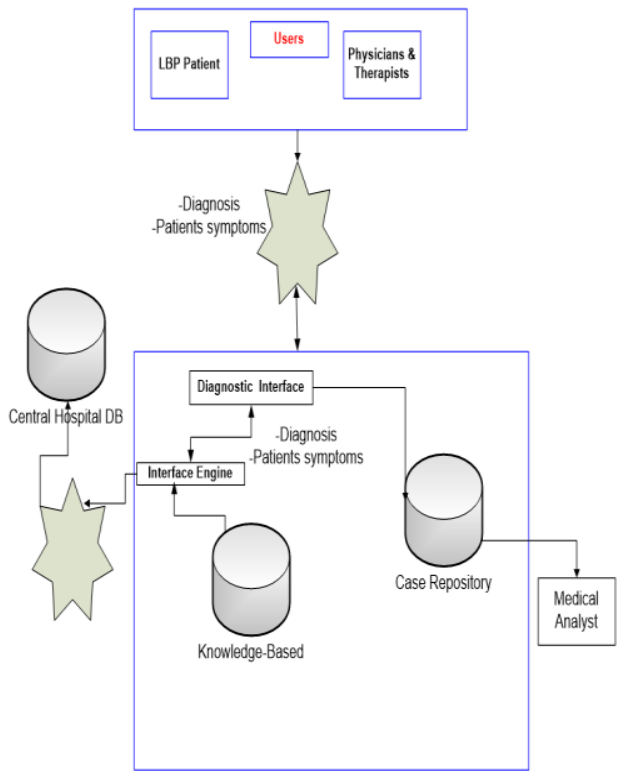

Figure. 4The WDSS diagnosis LBP

The above system works in simple way. It just provide the graphical interface to the patients as well as the physicians to access the website. Now, a patient can log in through the web-based interface to start a selfdiagnosis session without the help of a clinician's assistance. When the patients are log in the system they will get some predefines questions to answer to diagnosis and when they have done they can submit those question. Every time the patient $\log$ in the web site the diagnostic interface create a session information with the case repository database and all the information's are save for future use that can be used for medical analysts. When the patient submit the answer its go the inference engine and it generates a diagnosis that include one or more parts which refer to specific medical conditions, diseases or abnormalities. But before the inference engine generate a diagnosis it send a query to the central database to check the patient background which was saved in the database and compare with the present problems that patient submitted which will help to provide more accurate diagnosis but there is a question that it can access the central database or not which is depend on the mutual agreement with the care provider and the government. Then the clinician can check the proposed diagnosis and make some correction if he or she wants to. Here is the sample question that the patient has to answer.

\section{Result and discussion}

In this study, a Web-based Decision Support System is proposed to diagnose lower back pain patient. The WDSS normally applies for chronic LBP diagnosis (non-trauma), is it possible to apply for an acute low back pain diagnosed or accident/ trauma patient. This system uses a rule -based model for LBP diagnosis, any model is more effective than rule-based decision support. Medical Imaging utilization would more effective 
(with a certainty level), and a result in LBP diagnosis takes less time consuming than the existing system and for a certain diagnosis; whereas using medical imaging for LBP diagnosis, a result in a cost increased. According to test results, the usability score of scale is high, and the system is better. Moreover, the physicians pleased to the functionality of WDSS.

\section{Conclusion}

Clinical decision support system is a tool to help clinicians giving a faster and certain diagnosis and updating knowledge based from experts is very important as well. Improving a quality of medical care; STEEEP by giving the right treatment to the right person within a right time. Finally, we conclude that, the new web based system is easy to use framework and challenging in compare to the traditional system. The evaluation of web based LBP diagnosis is attend to knowledge base verification, system verification and clinical efficacy. It will reduce the medical error and can reduce the time for the patients. This system is totally patients perspective and it is safe for all user although one of the major barrier is it cannot provide and acute service.

\section{References}

[1]. Froseen SE, Corey AS, “ Clinical Decision Support and Acute Low Back Pain: Evidence-Based Order Sets.," Journal of American Colleague of Radiology. 2012; 9(10):704-712.

[2]. Murphy DR and Hurwitz EL, "Application of a diagnosis-based clinical decision guide in patients with low back pain.," Chiropractic \& Manual Therapies $2011 ; 19(26): 1-10$

[3]. Schoen DC. Low Back Pain. Orthopaedic Nursing 2004; 23(2): 154-157.

[4]. Evan RS. Case studies in clinical decision support: LDS Hospital Experience. In: Greenes RA. (eds.) Clinical Decision Support : The Road Ahead. Oxford: Elsevier Inc ; 2007.p.143-168.

[5]. Lin L, Hu PJ, Olivia R, Sheng L. A decision support system for lower back pain diagnosis: Uncertainty management and clinical evaluations. Journal of Decision Support Systems 2006; $42: 1152-1169$.

[6]. Wendy Oude Nijeweme - d'Hollosy1, Lex van Velsen1,2, Ilse C.S. Swinkels3 and Hermie Hermens1, " Clinical Decision Support Systems for Primary Care: The Identification of Promising Application areas and an Initial Design of a CDSS for lower back pain," 17th International Symposium on Health Information Management Research - ISHIMR 2015

[7]. Power, Daniel J., "Web-Based and Model-Driven Decision Support Systems: Concepts and Issues" (2000).AMCIS 2000 Proceedings. Paper 387.

[8]. Ercüment Güvenç, Gürcan Çetin , Gürbüz Akçay, "Development of a Web-based Decision Support System for Pediatric Patients," International Journal of Computer Applications (0975 - 8887) Volume 114 - No. 6, March 2015

[9]. Özata, M. and Aslan, Ş., 2004. Klinik Karar Destek Sistemleri ve Örnek Uygulamalar. The Medical Journal of Kocatepe, Vol 5, No $2,11-17$

[10]. Basilakis, J., Lovell, N. H., Redmond, S. J. and Celler, B. G., 2010. Design of a Decision-Support Architecture for Management of Remotely Monitored Patients. IEEE

[11]. Shim J.P., Warkentin M., Courtney J.F., Power D.J., Shards R., Carlsson Ch.: Past, Present and Future of Decision Support Technology. Decision Support Systems, Nr. 33, pp. 111-126, (2002)

[12]. Bonczek, R.H., Holsapple, C.W., Whinston, A.B.: Foundations of Decision Support Systems. Academic Press, New York (1981)

[13]. Turban, E.: Decision Support and Expert Systems: Management Support Systems. 4th Edition, Macmillan Publishing Company, New York (1995)

[14]. R. McLeod, G. Schell: Management Information Systems, 10th Edition, Prentice Hall, pp. 250-274 (2006).

[15]. S. French, M. Turoff: Decision Support

[16]. Systems, Communications of the ACM, Vol. 50, No 3, pp. 39-40 (2007).

[17]. Chien-Chih Yu: A Web-Based Consumer- Oriented Intelligent Decision Support System for Personalized E-Services, ACM International 